\title{
Association of apelin and AF in patients with implanted loop recorders undergoing catheter ablation
}

\author{
Uher $\mathrm{T}^{1}$, Bohm $\mathrm{A}^{1,2,3}$, Urban $\mathrm{L}^{2}$, Tothova $\mathrm{L}^{4,5}$, Bacharova $\mathrm{L}^{5,6}$, Musil $\mathrm{P}^{5}$, Kyselovic $\mathrm{J}^{5}$, \\ Michalek $\mathrm{P}^{1,3}$, Vachalcova $\mathrm{M}^{1,7}$, Olejnik $\mathrm{P}^{2,5}$, Hatala $\mathrm{R}^{2,3}$
}

Academy-Research Organization, Bratislava, Slovakia. tomasuher23@gmail.com

\begin{abstract}
BACKGROUND: Previous studies showed an association between apelin and atrial fibrillation (AF). The aim of this study was to analyse the effect of pulmonary vein isolation (PVI) in patients with paroxysmal AF on plasmatic apelin concentrations.

METHODS: Nine consecutive patients (aged from 43 to 69 years, 3 females and 6 males) with documented paroxysmal atrial fibrillation and implanted loop recorders (ILR) for continuous ECG monitoring were included in this study. All the patients underwent a radiofrequency catheter ablation with PVI.

RESULTS: The plasmatic concentration of apelin increased after PVI. The average plasmatic concentration of apelin before PVI was $0.299 \mathrm{ng} / \mathrm{ml}( \pm 0.16), 3$ months after PVI $0.462 \mathrm{ng} / \mathrm{ml}( \pm 0.10)$ and 9 months after PVI $0.565 \mathrm{ng} / \mathrm{ml}( \pm 0.146)$. There was an increase in the concentration of apelin 3 months and 9 months after the $P V I$ by $0.163 \mathrm{ng} / \mathrm{ml}(p=0.07)$ and by $0.266 \mathrm{ng} / \mathrm{ml}(p=0.01)$, respectively. The concentration of apelin inversely correlated with the AF burden $(r=-0.44, p=0.03)$.

CONCLUSIONS: Our study showed a significant increase in apelin levels after the reduction of AF burden via $\mathrm{PVI}$ and an inverse correlation with AF burden. Apelin might be a promising marker of AF (Tab. 2, Fig. 2, Ref. 28). Text in PDF www.elis.sk

KEY WORDS: apelin, atrial fibrillation, pulmonary vein, catheter ablation.
\end{abstract}

\section{Introduction}

Atrial fibrillation (AF) is the most common sustained arrhythmia. The estimated number of people with AF worldwide was more than 33 million in $2010(1,2)$. There is an expectation of an increase in $\mathrm{AF}$ prevalence due to a better detection of silent $\mathrm{AF}$, alongside with an increasing age and conditions predisposing to $\mathrm{AF}$. AF is associated with a significant morbidity and mortality and remains the major cause of strokes worldwide $(3,4)$. The only screening method is the standard 12-lead ECG, which bears several limitations especially in the case of subclinical AF. In these situations, a prolonged ECG monitoring is required. However, its high cost and inconvenience make this diagnostic method impractical (5,

\footnotetext{
${ }^{1}$ Academy - Research Organization, Bratislava, Slovakia, ${ }^{2}$ National Cardiovascular Institute, Bratislava, Slovakia, ${ }^{3}$ Faculty of Medicine, Slovak Medical University, Bratislava, Slovakia, ${ }^{4}$ Institute of Molecular Biomedicine, Faculty of Medicine, Comenius University, Bratislava, Slovakia, ${ }^{5}$ Faculty of Medicine, Comenius University, Bratislava, Slovakia, ${ }^{6}$ International Laser Center, Bratislava, Slovakia, and ${ }^{7}$ East-Slovak Institute of Cardiovascular Diseases, Kosice, Slovakia
}

Address for correspondence: T. Uher, MD, 1. maja 84/39, SK-901 01 Malacky, Slovakia.

Phone: +421914241391

Acknowledgements: An independent research grant of the Ministry of Education, Science, Research and Sport of the Slovak Republic (VEGA 1/0338/17). An independent research grant of the Ministry of Education, Science, Research and Sport of the Slovak Republic (KEGA 030UK-4/2019).
6). Therefore, there is an urgent need for a plasmatic biomarker with a significant predictive ability for AF.

Apelin is a peptide hormone produced especially in atrial cardiomyocytes that affects cardiovascular system by counterbalancing the renin-angiotensin-aldosterone axis, stimulating cardiac contractility and vasodilatation (7). There is also an evidence that apelin shortens the duration of action potential in atrial myocytes via its effect on multiple ionic channels (8). Several studies showed that apelin concentrations were significantly lower in patients with lone AF compared to the patients without AF (9). Current standard approach to assess the efficacy of PVI by means of 24-72 hour ECG monitoring lacks a sufficient reliability to exclude asymptomatic AF recurrence (10).

Our aim was to analyse apelin concentrations in plasma before and after the catheter ablation and to analyse the relation between the plasmatic levels of apelin and the AF burden quantified by the implantable loop recorders (ILR) in the patients with paroxysmal AF. We hypothesized that the concentration of apelin increases after the PVI.

\section{Material and methods}

\section{Patients and study protocol}

The study was designed as a prospective cohort study. Nine consecutive patients with symptomatic, paroxysmal AF and implanted ILR undergoing a radiofrequency catheter pulmonary veins 
isolation (PVI) in the Slovak National Institute of Cardiovascular Diseases were included.

The inclusion criteria were: age 18 or higher, planned PVI, implanted ILR at least 2 months before the PVI, symptomatic and drug refractory, paroxysmal AF characterized by a spontaneous termination or medical cardioversion within 7 days of onset documented prior to the ILR implantation, continuous, effective oral anticoagulation with warfarin (INR 2-3) at least one month prior to the PVI, ability and willingness of the patients to provide a written informed consent with the study, antiarrhythmic therapy with beta blocker and amiodarone started at least 3 months before ILR implantation and continued at least 3 months after the PVI.

Exclusion criteria included: supraventricular arrhythmia other than paroxysmal AF, previous catheter ablation, implanted pacemaker or ICD, anticipating major cardiac surgery within the course of this study, stable coronary artery disease functional class or history of acute coronary syndrome, heart failure, history of acute or chronic inflammatory disease, chronic kidney disease, left atrial thrombus detected by transoesophageal echocardiography prior to the PVI and uncontrolled hyperthyroidism.

Baseline clinical data were collected one day before the PVI. Information about the AF burden defined as the percentage of time spent in atrial fibrillation was downloaded from the ILR immediately after the procedure, 3 months and 9 months after the PVI. Peripheral blood samples were taken at the day of the PVI, 3 months and 9 months after the PVI

The study was approved by the ethical committee of the Slovak National Institute of Cardiovascular Diseases and a written informed consent was obtained from all the patients before the study inclusion.

\section{Radiofrequency catheter ablation with pulmonary veins isolation (PVI) and implantable loop recorder (ILR)}

These methods were described in our previous study (11).

\section{Biochemical analysis}

Peripheral fasting blood was taken in the morning into K3EDTA tubes. The blood was centrifuged at $2700 \mathrm{~g}$ for 5 minutes and the obtained plasma samples were stored at $-20^{\circ} \mathrm{C}$. The apelin- 12 concentration was measured using a commercially available ELISA kit (Phoenix Pharmaceutical, Karlsruhe, Germany) in serum samples. Fifty microliters of samples were used for measurement according to the protocol of the manufacturer.

\section{Statistical analysis}

Continuous variables are presented as the means and standard deviations, categorical variables are presented as percentages. The association between the variables was tested using the Spearman rank correlation coefficient $\rho$. Nonparametric Theil-Sen method was used to calculate a regression line displayed on the scatterplots. Mann-Whitney test was used to test differences in the study group before and after PVI. Data were analysed using StatsDirect statistical software
Tab. 1. Descriptive statistics of the study population. Continuous data are presented as the mean \pm standard deviation.

\begin{tabular}{lc}
\hline Baseline characteristics & Total $(\mathrm{n}=9)$ \\
\hline Age (years) & $56.9(43-69)$ \\
Female gender, no. $(\%)$ & $3(33.3 \%)$ \\
BMI $\left(\mathrm{kg} / \mathrm{m}^{2}\right)$ & $29.1 \pm 5$ \\
AF history before PVI (years) & $7.1 \pm 4.5$ \\
LA size at PVI (mm) & $44 \pm 4$ \\
Arterial hypertension, no. (\%) & $6(66.6 \%)$ \\
\hline
\end{tabular}

version 10.0.17 (http://www.statsdirect.com). p $<0.05$ was considered statistically significant.

\section{Results}

Our study population included 3 females and 6 males with the age ranged from 43 to 69 years. The most common comorbidity was arterial hypertension that occurred in $67 \%$ of the patients. All received an equal therapy with a cardio selective long acting beta-blocker (metoprolol or bisoprolol), amiodarone and warfarin, at least 3 months prior to the ILR implantation until the first follow-up ( 3 months after the PVI). If the AF burden at the first follow-up was less than $1 \%$, therapy with beta-blocker and amiodarone was terminated (Tab. 1).

The mean follow-up before the PVI was 63 days (ranging from 44 to 88 days), the mean follow-up 3 months after the PVI was 92 days (ranging from 84 to 112 days) and the mean followup 9 months after the PVI was 280 days (ranging from 201 to 307 days). In average, the relative AF burden decreased by $82 \%$ three months and by $78 \%$ nine months after the procedure (Tab. 2).

\section{Association between apelin before and after PVI}

The average plasmatic concentration of apelin before PVI was $0.299 \mathrm{ng} / \mathrm{ml}( \pm 0.166), 3$ months after PVI was $0.462 \mathrm{ng} / \mathrm{ml}( \pm 0.10)$ and 9 months after PVI was $0.565 \mathrm{ng} / \mathrm{ml}( \pm 0.146)$. The average difference between plasmatic concentration of apelin before PVI and plasmatic concentration after 3 months was $0.163 \mathrm{ng} / \mathrm{ml} ; 95 \%$ confidence interval $(\mathrm{CI}):-0.34$ to $0.02 ; \mathrm{p}=0.07$. The average difference between plasmatic concentration of apelin before PVI and plasmatic concentration after 9 months was 0.266 (CI: -0.44 to $-0.07 ; \mathrm{p}=0.01)$ (Fig. 1).

\section{Correlation between apelin and AF burden}

There was a statistically significant inverse correlation between apelin levels with corresponding AF burden before and after PVI (Figure 1; $\mathrm{r}=-0.44, \mathrm{p}=0.03$ ) (Fig. 2).

Tab. 2. Loop recorder characteristics. Data presented as the mean and (minimum, maximum range), or \pm standard deviation where appropriate.

\begin{tabular}{lccc}
\hline Variable & $\begin{array}{c}\text { Before PVI } \\
\text { (Baseline) }\end{array}$ & $\begin{array}{c}3 \text { months after } \\
\text { PVI }\end{array}$ & $\begin{array}{c}9 \text { months after } \\
\text { PVI }\end{array}$ \\
\hline Follow-up period (days) & $63(44-88)$ & $92.1(84-112)$ & $280(201-307)$ \\
AF burden, mean (\%) & $34.3(35)$ & $6.4(8.3)$ & $5.2(7.8)$ \\
AF burden, relative reduction (\%) & N/A & $82 \pm 15$ & $78 \pm 39$ \\
\hline
\end{tabular}




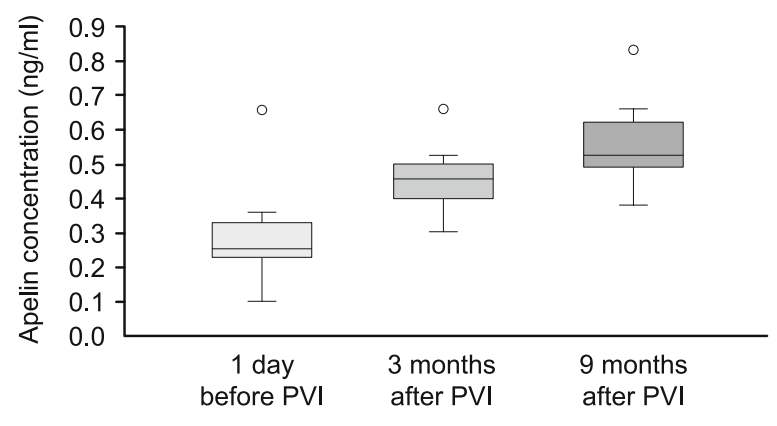

Fig. 1. Box and whisker plots.

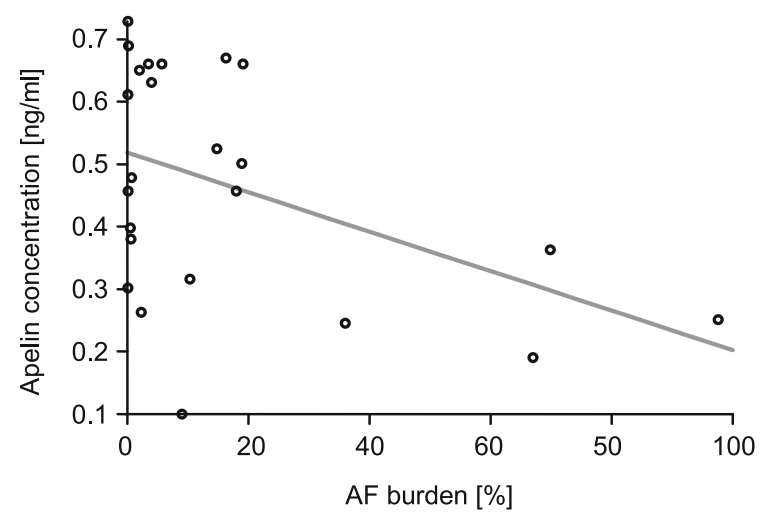

Fig. 2. Scatter plot of correlation between apelin levels with corresponding AF burden one day before, 3 months after and 9 months after pulmonary veins isolation, respectively.

\section{Discussion}

In this pilot study, we have demonstrated a significant inverse correlation between the plasma levels of apelin and decreased AF burden post PVI. Thus, our preliminary results suggest that low post-ablation apelin plasma levels are a possible marker for AF recurrences and these patients should be more extensively followed by extended ECG monitoring.

Apelin, an endogenous peptide ligand of the previously orphaned G-protein-coupled receptor APJ, is synthesized as a preproprotein, which is cleaved through $\mathrm{N}$-terminal proteolysis to generate several mature biologically active forms including apelin-36, apelin-17, apelin-16, apelin-13 and apelin-12 (12-14). Apelin is highly expressed in cardiomyocytes, endothelial cells, and vascular smooth muscle cells (VSMC), suggesting that apelin may play an important role in physiology and pathophysiology of cardiovascular system $(15,16)$. Recent studies showed an implication of apelin in AF. For example, decreased circulating levels of apelin were observed in lone AF patients and in patients with persistent $\mathrm{AF}(7,8)$.

Recent work has confirmed that apelin-12 is a powerful vasodilator and positive inotrope, and appears to be counter-regulated, at least to some extent, with the angiotensinogen pathway (17, 18). Apelin also may act centrally to counteract the vasopressin pathway in the regulation of extracellular fluid volume (19). The renin-angiotensin-aldosterone system (RAAS) signalling plays an important role in AF. Angiotensin II (ANG II) and AT1 are associated with the process of atrial electrical remodelling (20) and fibrosis (21). RAAS inhibition was considered as a cornerstone for the primary and secondary prevention of AF. Apelin has also an influence on electrophysiological function of atrial myocytes, shortening the action potential, which was demonstrated in vitro on animal model (22). Several studies showed that apelin has antiinflammation and antioxidative stress effects $(23,24)$. Apelin is a second catalytic substrate for angiotensin converting enzyme (ACE) 2 and functions as an inotropic and cardiovascular protective peptide (25). The increased level of ACE perhaps reduces the level of apelin. In addition, blockade of the renin-angiotensin system has been shown to reduce the incidence of AF, strengthening the case for a pivotal role for this humoral axis in this arrhythmia.

Reduced apelin levels in patients with "lone" AF, when compared to healthy population were reported previously (8). More recently, Wang YZ et al (2018) reported a higher apelin serum levels as a predictor of a recurrence-free successful outcome of PVI in $\mathrm{AF}$ patients (26). By demonstrating the increase in plasmatic level of apelin after reducing the AF burden via PVI, our findings suggest that the relationship could be causal. We designed a pilot study, where for the first time, apelin was examined in patients with implanted loop recorders undergoing catheter ablation of AF. Furthermore, an inverse correlation between apelin levels and AF burden adds a new evidence that apelin is not only associated with the presence or absence of AF, but also with the amount of AF. These two findings from our pilot study suggest that apelin might be a promising biomarker of AF, which deserves further research.

The current standard approach to assess the efficacy of PVI by means of 24-72 hour ECG monitoring lacks a sufficient reliability to exclude asymptomatic AF recurrences. Long-term ECG monitoring techniques e.g. implantable loop recorders or ECG patches are available, however, at both higher costs and inconvenience for the patient $(27,28)$. Plasmatic biomarkers like apelin might identify patients with a high risk of AF recurrence that would benefit from prolonged ECG monitoring. This would be especially useful in screening the patients after PVI, where recurrent, asymptomatic paroxysms of AF are common.

Our pilot study confirmed that apelin had a potential of becoming a plasmatic biomarker for $\mathrm{AF}$, but further research focused on apelin sensitivity and specificity is needed to validate its use in clinical practice.

\section{Limitations}

The major limitation of our study was the sample size. However, this was a pilot study to test the hypothesis that the concentration of apelin increases after the PVI, to evaluate the potential role of apelin as a biomarker of AF and to support further research in this area.

Our study showed a relationship between AF and apelin level. However, as only patients without heart failure were included, it is not known whether apelin level reflects AF per se or increased strain in the LA. 


\section{Conclusion}

Our pilot study showed a significant increase in apelin levels after a reduction of AF burden after PVI and also an inverse correlation between apelin levels and AF burden in patients with paroxysmal AF. Therefore, apelin appears to be a promising biomarker of atrial fibrillation especially in patients undergoing PVI, but more extensive studies are needed to focus on the potential for future use in the clinical practice.

\section{References}

1. Kirchhof P, Benussi S, Kotecha D et al. 2016 ESC Guidelines for the management of atrial fibrillation developed in collaboration with EACTS. Eur J Cardiothorac Surg 2016; 50 (5): e1-e88.

2. Cavaco DM. Epidemiology of atrial fibrillation in Galicia: Insights from 'big data'. Rev Port Cardiol 2019; 38 (1): 31-32.

3. Kannel WB, Abbott RD, Savage DD et al. Epidemiologic features of chronic atrial fibrillation: the Framingham study. N Engl J Med 1982; 306 (17): 1018-1022.

4. Rodríguez-Mañero M, López-Pardo E, Cordero-Fort A et al. Prevalence and outcomes of atrial fibrillation in a European healthcare area gained through the processing of a health information technology system. Rev Port Cardiol 2019; 38 (1): 21-29.

5. Freedman B, Camm J, Calkins $\mathbf{H}$ et al. AF-Screen Collaborators. Screening for atrial fibrillation: a report of the AF-SCREEN International Collaboration. Circulation 2017; 135: 1851-1867.

6. Young B. New standards for ECG equipment. J Electrocardiol $2019 \mathrm{Jul}$ 25. pii: S0022-0736 (19)30394-2. doi: 10.1016/j.jelectrocard.2019.07.013.

7. Chong KS, Gardner RS, Ashley EA et al. Emerging role of the apelin system in cardiovascular homeostasis. Biomark Med 2007; 1 (1): 37-43.

8. Ellinor PT, Low AF, Macrae CA. Reduced apelin levels in lone atrial fibrillation. Eur Heart J 2006; 27 (2): 222-226.

9. Cheng CC, Weerateerangkul P, Lu YY et al. Apelin regulates the electrophysiological characteristics of atrial myocytes. Eur J Clin Invest 2013; 43 (1): 34-40.

10. Bou Ezzeddine H, Vachulova A, Svetlosak M et al. Occurrence of symptoms after catheter ablation of atrial fibrillation. Bratisl Lek Listy 2015; 116 (8): 461-464.

11. Böhm A, Tothova L, Urban L, Slezak P, Bacharova L, Musil P, Hatala $\mathbf{R}$. The relation between oxidative stress biomarkers and atrial fibrillation after pulmonary veins isolation. J Electrocardiol 2016; 49 (3): 423-428.

12. Chandrasekaran B, Dar O, McDonagh T. The role of apelin in cardiovascular function and heart failure. Eur J Heart Fail 2008; 10: 725-732.

13. O'Carroll AM, Lolait SJ, Harris LE et al. The apelin receptor APJ:journey from an orphan to a multifaceted regulator of homeostasis. J Endocrinol 2013; 219: R13-35.
14. Pisarenko OI, Serebryakova LI, Studneva IM et al. Effects of structural analogues of apelin-12 in acute myocardial infarction in rats.J Pharmacol Pharmacother 2013; 4: 198-203.

15. Gardner RS. Nongenetic markers in heart failure. Biomark Med 2014; 8 (6): 773-775.

16. Hashimoto $\mathbf{T}$, Kihara $\mathbf{M}$, Ishida $\mathbf{J}$ et al. Apelin stimulates myosin light chain phosphorylation in vascular smooth muscle cells. Arterioscler Thromb Vasc Biol 2006; 26: 1267-1272.

17. Tatemoto K, Takayama K, Zou MX et al. The novel peptide apelin lowers blood pressure via a nitric oxide-dependent mechanism. Regul Pept 2001; 99: 87-92.

18. Ishida J, Hashimoto T, Hashimoto $Y$ et al. Regulatory roles for APJ, a seven-transmembrane receptor related to angiotensin-type 1 receptor in blood pressure in vivo. J Biol Chem 2004; 279: 26274-26279.

19. De Mota N, Lenkei Z, Llorens-Cortes C. Cloning, pharmacological characterization and brain distribution of the rat apelin receptor. Neuroendocrinology 2000; 72: 400-407.

20. Boldt A, Wetzel U, Weigl J et al. Expression of angiotensin II receptors in human left and right atrial tissue in atrial fibrillation with and without underlying mitral valve disease. J Am Coll Cardiol 2003; 42: 1785-1792.

21. Kupfahl C, Pink D, Friedrich $\mathrm{K}$ et al. Angiotensin II directly increases transforming growth factor beta1 and osteopontin and indirectly affects collagen mRNA expression in the human heart. Cardiovasc Res 2000; 46: 463-475.

22. Cheng CC, Weerateerangkul P, Lu YY et al. Apelin regulates the electrophysiological characteristics of atrial myocytes. Eur J Clin Invest 2013; 43 (1): 34-40.

23. Zhou Q, Cao J, Chen L. Apelin/APJ system: a novel therapeutic target for oxidative stress-related inflammatory diseases (Review). Internat J Mol Med 2016; 37 (5): 1159-1169.

24. Bircan B, Cakir M, Kırbag S et al. Effect of apelin hormone on renal ischemia/reperfusion induced oxidative damage in rats. Renal Failure 2016; 38 (7): 1122-1128.

25. Chen LJ, Xu R, Yu HM et al. The ACE2/apelin signaling, microRNAs, and hypertension. Internat J Hypertension 2015, Article ID 896861, 6 pages.

26. Wang YZ, Fan J, Zhong B et al. Apelin: A novel prognostic predictor for atrial fibrillation recurrence after pulmonary vein isolation. Medicine (Baltimore) 2018; 97 (39): e12580.

27. Ackermans PAJ, Solosko TA, Spencer et al. A user-friendly integrated monitor-adhesive patch for long-term ambulatory electrocardiogram monitoring. J Electrocardiol 2012; 45 (2): 148-153.

28. Wachter R, Groschel K, Gelbrich G et al. Find-AF (RANDOMISED) Investigators and Coordinators. (2017). Holter-electrocardiogram-monitoring in patients with acute ischaemic stroke (find-AFRANDOMISED): An open-label randomised controlled trial. Lancet Neurol 2017; 6 (4): 282-290. 